\title{
Ascitis fetal como forma de presentación de agenesia de la vena cava inferior
} Fetal ascites as clinical presentation of inferior vena cava agenesis

\author{
Dr. Pedro L. Mercado, ${ }^{a}$ Dr. Daniel H. Liberto, ${ }^{a}$ Dra. Julia Udaquiola, ${ }^{a}$ Dr. Patricio Cieri, ${ }^{a}$ \\ Dr. Roberto L. Vagni, ${ }^{a}$ Dr. Pablo Lobos ${ }^{a}$ y Dr. Juan M. Moldes Larribas ${ }^{a}$
}

\section{RESUMEN}

La vena cava inferior (VCI) está constituida por tres segmentos de diferente origen embriológico. De su mala fusión, surge un amplio espectro de anomalías. La prevalencia de anomalías de la VCI es de 0,07-8,7\% de la población. Generalmente, se diagnostica como hallazgo incidental en la vida adulta. Representa el 5-9,5\% de las trombosis venosas profundas idiopáticas en menores de 30 años sin factores de riesgo asociados.

Se presenta a una recién nacida a término con diagnóstico prenatal de ascitis en la semana 20 de gestación. Se diagnosticó, mediante angiotomografía abdominal, la agenesia de VCI. El tratamiento de pacientes con agenesia de la VCI se basa en el manejo de las complicaciones. Debido al mayor riesgo que presentan de sufrir un evento trombótico, se debe considerar la profilaxis antitrombótica a largo plazo. Se recomienda iniciar profilaxis anticoagulante en la pubertad.

Palabras clave: ascitis fetal, agenesia, vena cava inferior, pediatría, malformaciones vasculares.

\begin{abstract}
Inferior Vena Cava (IVC) is composed of three segments from different embryological origin. Its lack of fusion originates a wide spectrum of anomalies of the IVC. These malformations are present in $0.07-8.7 \%$ of the population. It is generally diagnosed as an incidental finding in adult life. It represents between 5 and $9.5 \%$ of idiopathic deep vein thrombosis in patients younger than 30 years old without associated risk factors.

We present a case of a term newborn with prenatal diagnosis of ascites during the $20^{\text {th }}$ week of gestation. IVC Agenesis was diagnosed with the use of abdominal angiotomography.

The treatment of patients with IVC Agenesis is based on the management of its complications. Due to the increased thrombotic risk of these patients, we should consider lifelong anticoagulation. We suggest initiating it during puberty. Key words: fetal ascites, agenesis, inferior vena cava, pediatric, vascular malformations.
\end{abstract}

a. Servicio de Cirugía y Urología Pediátrica, Hospital Italiano de Buenos Aires, Ciudad Autónoma de Buenos Aires, Argentina.

Correspondencia:

Dr. Pedro L. Mercado: pedro.mercado@hospitalitaliano.org.ar

Financiamiento: Ninguno.

Conflicto de intereses: Ninguno que declarar.

Recibido: 1-11-2017

Aceptado: 07-2-2018 http:/ / dx.doi.org/10.5546/aap.2018.e621

Cómo citar: Mercado PL, Liberto DH, Udaquiola J, et al. Ascitis fetal como forma de presentación de agenesia de la vena cava inferior. Arch Argent Pediatr 2018;116(4):e621-e625.

\section{INTRODUCCIÓN}

Las malformaciones de la vena cava inferior (VCI) constituyen un grupo de anomalías del desarrollo embrionario poco frecuentes en la población general. Dentro de estas, se encuentra la agenesia de vena cava inferior (AVCI), con una frecuencia de $0,07 \%$ en la población. Si bien se trata de una patología de muy baja prevalencia, es la causante del $5 \%$ al 9,5\% de las trombosis venosas profundas (TVP) idiopáticas en pacientes menores de 30 años. ${ }^{1}$

Se presenta el caso de una paciente con diagnóstico prenatal de ascitis en la cual, luego de descartarse las causas más frecuentes de esta, se diagnosticó posnatalmente AVCI.

El objetivo del presente trabajo es identificar la AVCI como diagnóstico diferencial en pacientes con ascitis de presentación fetal o perinatal. Este hecho permitirá realizar un diagnóstico precoz y prevenir las complicaciones asociadas.

\section{CASO CLÍNICO}

Paciente de sexo femenino con diagnóstico ecográfico prenatal de ascitis a las 20 semanas de gestación (Figura 1). Madre primigesta sin antecedentes de relevancia. Se descartó hidrops mediante el seguimiento ecográfico seriado y se evidenció ascitis aislada moderada/grave sin cambios hasta el nacimiento. Presentó cariotipo fetal normal. Fue una recién nacida pretérmino de 36 semanas por cesárea, con $2700 \mathrm{~g}$ (percentilo 10), sin sintomatología cardiorrespiratoria, que no requirió oxígeno suplementario. $\mathrm{Al}$ nacer, se evidenció un abdomen marcadamente distendido, tenso, no doloroso, con matidez ante la percusión y signos de circulación colateral sobre la pared 
abdominal anterior. El resto del examen físico no presentó particularidades destacables.

Se realizaron los siguientes estudios complementarios:

- Estudios de laboratorio normales. Serologías negativas.

- Radiografía toracoabdominal, donde se evidenciaron ambos campos pulmonares sin signos de derrame pleural y silencio abdominal, compatible con ascitis (Figura 2).

- Ecografía abdominal con doppler hepático, que informó gran cantidad de líquido libre en la cavidad abdominal, ligera hepatomegalia con flujos hepáticos conservados, sin esplenomegalia.

- Ecocardiograma con evidencia de doble sistema vena cava superior (vena cava superior izquierda persistente que drenaba en el seno coronario dilatado).

- Se realizó una paracentesis diagnóstica con líquido abdominal compatible con trasudado -glucosa: $63 \mathrm{mg} / \mathrm{dl}$; proteínas: 3,9 mg/dl; triglicéridos: $41 \mathrm{mg} / \mathrm{dl}$; urea: $20 \mathrm{mg} / \mathrm{dl}$-. El análisis citológico del líquido mostró 160 células $(70 \%$ de mononucleares, $30 \%$ de polimorfonucleares).

Al tercer día de vida, se inició la alimentación enteral con leche con bajo contenido de grasas enriquecida con ácidos grasos de cadena mediana por gastroclisis, con buena tolerancia. Se aumentó progresivamente hasta alcanzar los requerimientos totales, sin presentar intercurrencias.

Ante la sospecha de una anomalía vascular, se realizó una flebotomografía computada, que

FIGURA 2. Radiografía toracoabdominal de frente

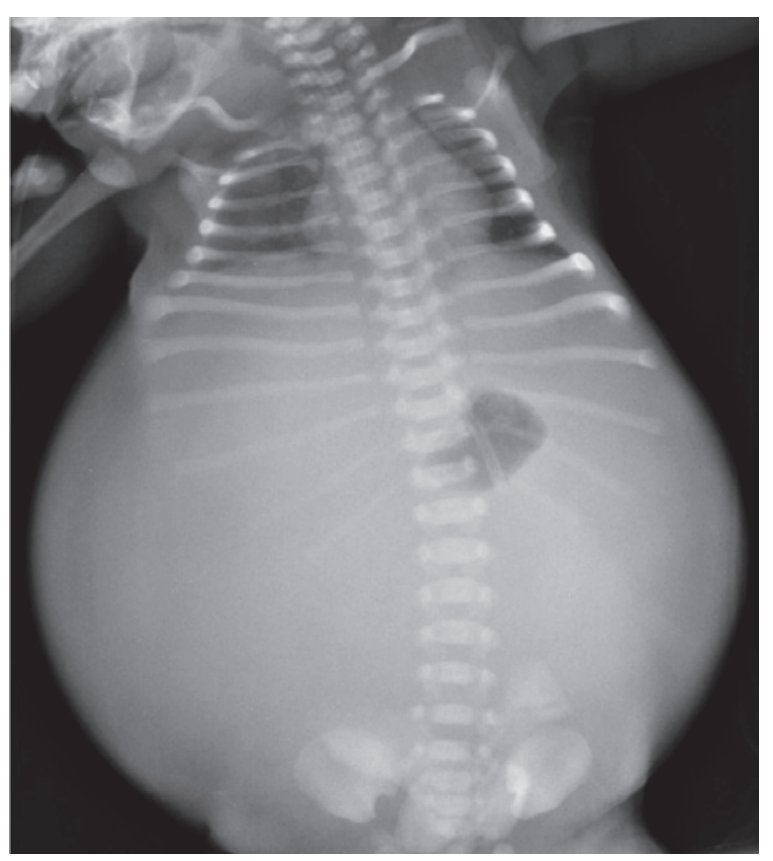

Se observa silencio abdominal, con presencia de gran cantidad de líquido (ascitis).
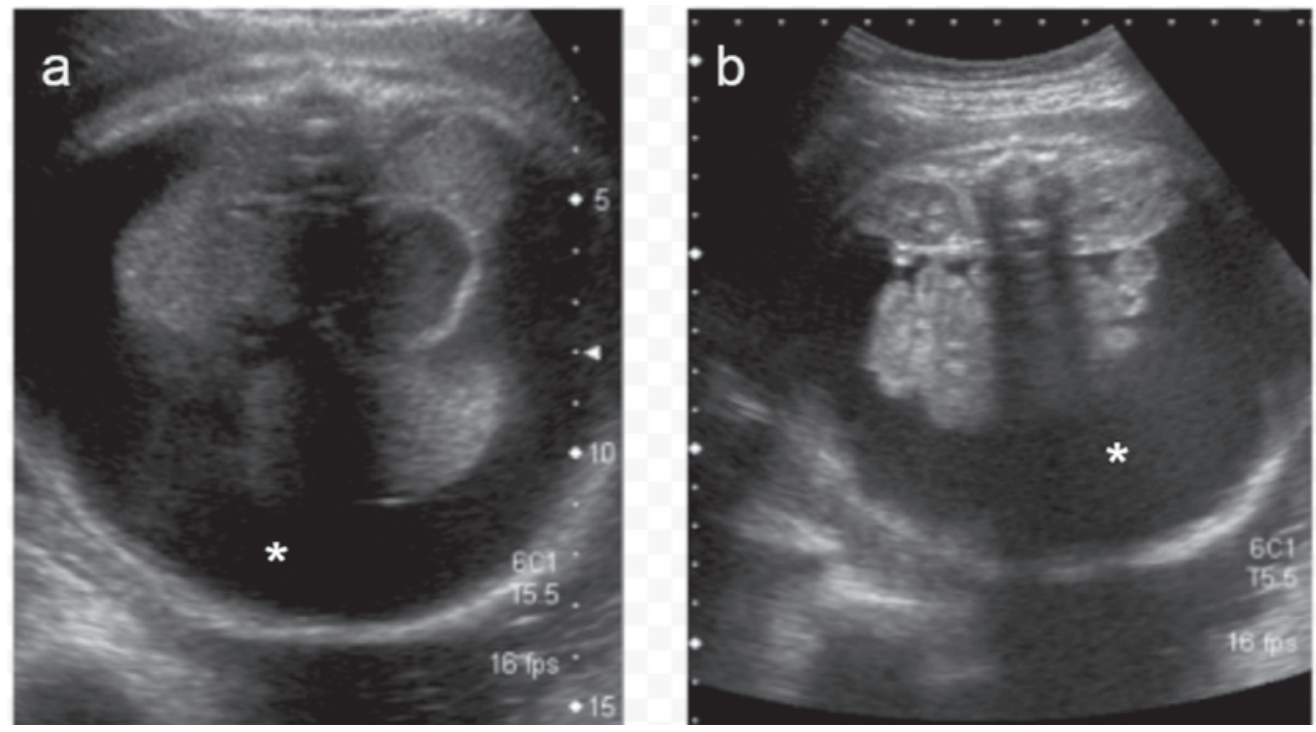

(a) Corte axial a nivel abdominal superior y (b) a nivel de los riñones, donde se observa abundante líquido libre abdominal $(*)$. 
mostró ausencia de permeabilidad de la VCI infrarrenal y de ambas venas ilíacas primitivas. El segmento suprarrenal de la VCI hasta su desembocadura en la aurícula derecha, por un lado, y las venas ilíacas externas y femorales, por el otro, se encontraban permeables (Figura 3). No se evidenciaron signos de trombosis ni otras malformaciones asociadas.

Una vez establecido el diagnóstico de AVCI, se decidió progresar la alimentación hasta lograr el aporte enteral completo. Permaneció internada para la recuperación nutricional, y se resolvió lentamente la ascitis. Fue dada de alta a los 3 meses de vida.

La paciente tiene actualmente 4 años, evoluciona asintomática, creciendo en el percentilo 75-90 de peso y en el percentilo 75 de talla. Se encuentra en seguimiento por la Clínica de Anomalías Vasculares de nuestra Institución, sin profilaxis antitrombótica al momento.

\section{DISCUSIÓN}

Se denomina "ascitis" a la presencia de líquido libre en la cavidad peritoneal. Puede ser un signo aislado (ascitis fetal aislada) ${ }^{2}$ o formar parte de una hidropesía fetal, con acumulación de líquido en, al menos, dos compartimentos fetales (derrame pericárdico, derrame pleural, ascitis, edema subcutáneo). Es causada por diferentes mecanismos, como drenaje linfático anómalo, obstrucción del retorno venoso por alguna masa extrínseca, insuficiencia cardíaca o hepática, disminución de la presión oncótica plasmática fetal, obstrucción del tracto urinario, aumento de la permeabilidad capilar o peritonitis meconial. ${ }^{3}$

Una vez confirmada la ascitis, es fundamental hacer el diagnóstico de su etiología para así poder instaurar un tratamiento adecuado y evitar potenciales complicaciones. En el caso clínico en cuestión, luego de descartar las causas más frecuentes de ascitis fetal, se llegó al raro diagnóstico de AVCI. ${ }^{4}$

La VCI se desarrolla entre las semanas $6^{a} y$ $8^{\text {va }}$ de gestación e involucra un complejo proceso de formación, regresión y fusión de tres pares de venas embrionarias de disposición longitudinal. Estas venas son las siguientes:

1. Venas cardinales posteriores.

2. Venas subcardinales.

3. Venas supracardinales.

La VCI definitiva está conformada por cuatro segmentos, que son, en orden cefalocaudal, intrahepático, suprarrenal, renal e infrarrenal. ${ }^{5}$

Una anomalía de la VCI refleja una regresión o persistencia anormal de las venas embrionarias. Esto puede deberse tanto a una alteración en el período de embriogénesis como a trombosis en el período prenatal. Se pueden producir muchas variaciones en su configuración anatómica, tales como las siguientes:

- Vena renal izquierda retroaórtica.

- Vena renal izquierda circumaórtica.

- Doble VCI infrarrenal.

- VCI infrarrenal izquierda.

- Continuación de la VCI con la ácigos o hemiácigos.

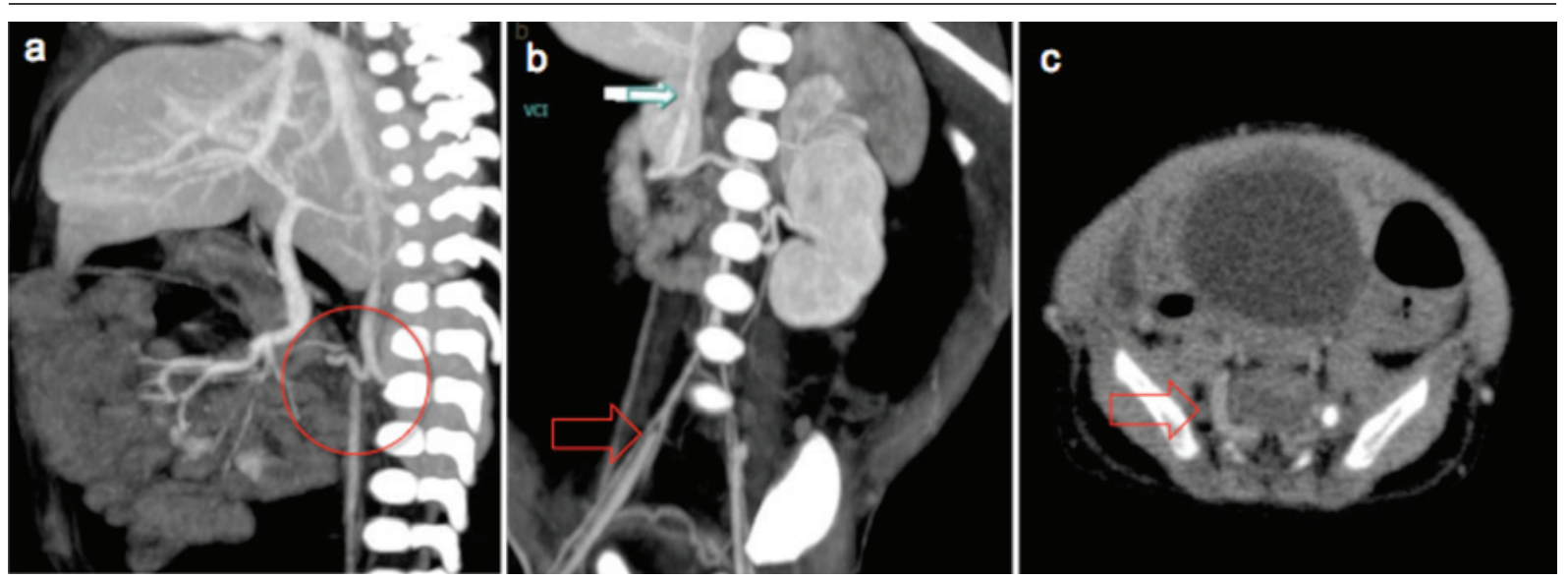

(a) Corte sagital y (b) corte coronal: ausencia de opacificación de la vena cava inferior por debajo de las venas renales (círculo) hasta las venas ilíacas externas (flecha). (c) Corte axial: se observa circulación colateral a través del plexo vertebrolumbar dilatado (flecha) 
- Ausencia de la VCI: ${ }^{5}$ suprarrenal, infrarrenal o de toda la VCI.

La ausencia de la VCI infrarrenal implica una falla en el desarrollo de las venas cardinales y supracardinales posteriores. ${ }^{6}$ Las malformaciones en la VCI están presentes en un 0,07-8,7\% de la población general. ${ }^{7}$

El sistema linfático comienza su desarrollo hacia fines de la quinta semana, aproximadamente, dos semanas más tarde que el sistema cardiovascular. Aún no está claro su origen, pero se cree que podrían formarse a partir del mesénquima in situ o a partir de evaginaciones saculares provenientes del endotelio de las venas. ${ }^{8}$ En la AVCI, al no haber un correcto desarrollo del sistema venoso, tampoco lo hay del linfático. La falta de drenaje linfático normal hacia el torrente sanguíneo podría ser la causante de la ascitis, como en el caso de nuestra paciente. La ascitis se resuelve espontáneamente cuando el retorno venoso compensa el sistema linfático anómalo gracias al desarrollo de circulación colateral (plexo paravertebral). De esta forma, la sangre de la porción caudal del cuerpo llega al corazón a través de la vena ácigos y la vena cava superior.

La prevalencia de AVCI es del 0,005\% al $1 \%$ en la población general. ${ }^{7}$ Habitualmente, es asintomática debido a la presencia de circulación colateral. Su diagnóstico se hace como un hallazgo de una masa torácica o paraespinal en imágenes, que corresponde a los sistemas venosos colaterales dilatados. ${ }^{9}$ Una forma de presentación, no menos importante en la población pediátrica o adulta joven, es la TVP, que representa entre el $5 \%$ y el 9,5\% de las TVP idiopáticas en menores de 30 años. ${ }^{1}$ Otras manifestaciones son dolor lumbar, tromboembolismo pulmonar, edema, dolor o várices de miembros inferiores, síndrome de compresión de la cola de caballo y varicocele bilateral. ${ }^{10-14}$ Aunque hay muchas causas posibles de ascitis fetal, la AVCI debe ser incluida entre los diagnósticos diferenciales, debido a sus repercusiones a futuro.

El tratamiento de pacientes con AVCI se basa en el manejo de las complicaciones. Debido al mayor riesgo que presentan los pacientes con malformaciones de la VCI de sufrir un evento trombótico, se debe considerar la profilaxis antitrombótica a largo plazo. Se debe alertar a los pacientes sobre situaciones que aumenten el riesgo de trombosis, tales como el esfuerzo físico inusual, la cirugía, la inmovilización prolongada o el uso de anticonceptivos orales. ${ }^{11}$
No existe un consenso respecto al manejo de las complicaciones trombóticas de los pacientes con malformaciones de la VCI. En el momento agudo, es necesaria la anticoagulación, aunque la duración del tratamiento no está establecida. En los casos de TVP asociados a anomalías de la VCI, la mayoría de los autores opta por la anticoagulación profiláctica de por vida y se basa en el riesgo de recurrencia de la TVP. ${ }^{15}$

En el caso que se presenta, se adoptó una conducta conservadora, que consistió en un seguimiento multidisciplinario por pediatras, hematólogos y especialistas en anomalías vasculares. Se decidió el manejo expectante con respecto al inicio de la anticoagulación dada la edad de la paciente. Se considera recomendable iniciar profilaxis o tratamiento anticoagulante en la pubertad o, excepcionalmente, si ocurre un evento trombótico previo.

\section{CONCLUSIÓN}

Muchos son los diagnósticos que se deben descartar ante la presencia de ascitis fetal o neonatal, pero no se puede dejar de pensar en las anomalías vasculares como sus causantes. El conocimiento de dicha afección por parte del paciente y su médico de cabecera es crucial para indicar la profilaxis adecuada y prevenir potenciales complicaciones a futuro.

\section{REFERENCIAS}

1. Rodríguez Lorenzo L, Rizza Siniscalchi N, Madrazo González Z, et al. Tratamiento de la trombosis venosa profunda asociada a agenesia de vena cava inferior. Revisión de las anomalías de vena cava inferior. Angiología 2011;63(6):254-60.

2. López MF, Chávez $\mathrm{S}$, Hidalgo $\mathrm{R}$, et al. Ascitis fetal aislada idiopática: caso clínico. Rev Chil Obstet Ginecol 2012;77(4):301-5.

3. Favre R, Dreux S, Dommergues $M$, et al. Nonimmune fetal ascites: A series of 79 cases. Am J Obstet Gynecol 2004;190(2):407-12.

4. De Sarasqueta P. Ascitis Neonatal. En Martínez Ferro M, Cannizzaro C, Rodríguez S, et al. Neonatología Quirúrgica. Buenos Aires: Grupo Guía; 2003.Págs.687-690.

5. Morató López AE, Quiróz Castro O, Ramírez Arias JL. Anomalías congénitas de la vena cava inferior. El rol de la tomografía computada multidetector. An Radiol Méx 2012;11(2):104-13.

6. Kim SS, Shin HC, Hwang JA, et al. Various congenital anomalies of the inferior vena cava: review of cross-sectional imaging findings and report of a new variant. Abdom Radiol (NY) 2017 [Epub ahead of print].

7. Saltz Gensas C, Martins Pires L, Lapa Kruse M, et al. Agenesis of the Inferior Vena Cava. Rev Bras Cardiol Invasiva 2012;20(4):427-30.

8. Sadler TW.Sistema cardiovascular. En Sadler TW.Langman. Embriología Médica. 8. ${ }^{\text {va }}$ ed. Buenos Aires: Panamericana; 2001.Págs.239-51. 
9. Milner LB, Marchan R. Complete absence of the inferior vena cava presenting as a paraspinous mass. Thorax 1980;35(10):798-800.

10. Langer F, Dos Santos D, Suertegaray G, et al. Bilateral Deep Vein Thrombosis Associated with Inferior Vena Cava Agenesis in a Young Patient Manifesting as Low Back Pain. Acta Méd Port 2017;30(4):333-7.

11. Cho BC, Choi HJ, Kang SM, et al. Congenital absence of inferior vena cava as a rare cause of pulmonary thromboembolism. Yonsei Med J 2004;45(5):947-51.

12. Tarango C, Kumar R, Patel M, et al. Inferior vena cava atresia predisposing to acute lower extremity deep vein thrombosis in children: A descriptive dual-center study. Pediatr Blood Cancer 2017;65(5):e26785.
13. Oterdoom DL, De Jong BM, Hoogland P, et al. Transient cauda equina compression syndrome and headache caused by internal vertebral venous plexus engorgement in a teenage female with vena cava inferior agenesis and iliac vein thrombosis. J Neurol Neurosurg Psychiatry 2007;78(11):1283-4.

14. Koc Z, Oguzkurt L. Interruption or congenital stenosis of the inferior vena cava: Prevalence, imaging, and clinical findings. Eur J Radiol 2007;62(2):257-66.

15. Martínez-Aguilar E, Medina-Maldonado FJ, Flórez González A, et al. Hipoplasia de la vena cava inferior asociada a una trombosis venosa profunda de los miembros inferiores. Angiología 2008;60(1):55-9. 